\title{
A Survey on Image Fusion Techniques for Image Enhancement in Digital Image Processing
}

\author{
Rajvinder Kaur \\ Department of Computer Science and Engineering \\ BBSBEC, Fatehgarh Sahib, Punjab, India
}

\author{
Rupinder Kaur \\ Professor \\ Department of Computer Science and Engineering \\ BBSBEC, Fatehgarh Sahib, Punjab, India
}

fusion methods are divided into two parts, that are:-

\section{Spatial Domain \\ 2. Frequency Domain}

\begin{abstract}
Image fusion procedure is helpful to attain a single output image that contains required data or information by merging two distorted input images. Image fusion is done by pulling out all the necessary information from two images or more than two images after which the extorted information is combined into a distinct fused image. This fused image has improved superiority as compare to the input images. Image fusion is prepared by implementing particular techniques. Those particular schemes for image fusion are introduced in this paper. This paper offers an overview to the work that has been done by in past.
\end{abstract}

\section{General Terms}

Image Enhancements, Image Fusion Techniques, Digital image processing.

\section{Keywords}

Image Fusion, Image Enhancements, Transform Domain, Spatial Domain, Frequency Domain.

\section{INTRODUCTION}

Every domain is transformed to digitalization with the improvement in technology. Consequently, with the more number of users leads to the initiation in troubles and errors in technology. So the solution to one of these troubles is the image fusion [1]. The main principle of image fusion is to conserve the enhanced part of the image. By merging several images, the significant data of the information can be preserved. On the basis of the input images, no restriction can be forced as it can be multi focus (focus more than one object) or multi sensor etc. Thus, the images like multi focus or multisensory can lead to the various issues like there is a probability that to cover multiple objects in the image it might avoid any informative part or object. Thus, the concept of image fusion comes to the existence. The image fusion refers to the process of combining multiple images with different focused objects, so that a single meaningful image can be created. The image fusion is applied in case when users have multiple images but those images lacks to capture all the objects in appropriate manner. All that images will consume large memory space that does not attain the significant information individually. The only solution to all these troubles is generated in the form of image fusion. In the procedure of image fusion, the two images are introduced as input and by applying fusion scheme these input images are combined into a particular image that contains the whole meaningful and real(actual) data of the image [2]. So this is how the image fusion resolves the trouble of more memory area consumption and partial insignificant images.

\section{TECHNIQUES}

To advance the superiority of digital images the image fusion schemes are used without corrupting its features. The image
Spatial domain methods are used to treat with the pixels in an image that pixel standards are changed to present the advancements in the image. So these types of image fusion methods are:-
a. Brovey Method
b. Principal Component Analysis
c. HIS based Method

Firstly, the image is changed to the frequency domain in the frequency domain method. In this method the Fourier transform is calculated after that other functions are implemented to it. In turn the inverse Fourier transform is calculated to attain the output image [3]. To enrich the superiority of the image the advancement is prepared by making few amendments to its data. The adjustments brightness, contrast, allocation of the grey level etc are feasible to execute.

\subsection{Principal Component Analysis}

The method which alters the number of related variables into the format of data set of unrelated variable is known as PCA is conversion method. In case of image compression and image classification it functions effectively and efficiently. With the aid of mathematical calculations and functions the alteration of related variables is done that guides it to the brief and best explanation of data set. The function of PCA is employed in the figure of principal component. The first major component inspected much of the disputes in the accessible information as flexible [4]. The second major component that is perpendicular to first component is loaded in the subspace. Then as a third component the importance of utmost discrepancy is chosen. The PCA is another method which can be applied in place of IHS technique.

\subsection{Discrete Cosine Transform}

The major attribute of Discrete Cosine Transform based on the coefficient values assists the better signal estimation. This practice is applied by many paradigms for renovating the image and also in resulting to watermarking. The main enhancement of this technique is that it has quick processing rate as contrast to other methods. This is the simple and lossless compression scheme [5].

In this the image is divided into two elements like spectral sub elements. The functioning of this system is quite identical to DFT i.e. Discrete Fourier Transformation modifies the image from spatial domain to frequency domain. 


\subsection{Discrete Wavelet Transform}

Discrete wavelet system is used to provide discrete wavelet depiction by altering the discrete signals. In this method, the image is decomposed into coefficients and then this coefficient evaluates with the threshold. If the coefficient value is lower than threshold then the zero value will be assigned to it. And if coefficient value is above the threshold significance are prearranged with the loss less compression method. DWT occupies division, threshold detail coefficients and then rebuild.

\subsection{Stationery Wavelet Transformation}

The Stationery Wavelet Transformation technique (SWT) is created to conquer the limitations of Discrete Wavelet Transformation method. The difficulty found in DWT was deficiency of conversion Invariance the resolution of this is to abolish the down samplers and up samplers in DWT [6]. The up sampling is offered on the filter coefficients in the $j^{\text {th }}$ level of paradigm by executing the following equation:

$$
2^{(j-1)}
$$

SWT is a superfluous method because the number in every level of output is identical number in corresponding input which guides to $\mathrm{N}$ number of redundancy corresponding to the $\mathrm{N}$ number wavelet coefficients."Algorithm of Trous" is another name of SWT that is derived from French a phrase that refers to the addition of zeros at filters. The "Trous" means holes that was generated by holschneider and named as un-decimated wavelet transform or paradigm of trous. SWT is an improved or adapted edition of DWT that does not devastate coefficient at each transformation level.

\subsection{Laplacian Pyramid}

LP functions on the basis of pyramid structure which includes the fundamentals like blocks that are related to the several stages of the actual image. The disintegration of image into a range of levels is completed by employing low pass filters which recursively filters the low level image. As the level increases the image becomes small by preceding the length of the image [7].

\subsection{IHS Fusion Technique}

The most commonly known fusion method for sharpening is the IHS technique. It is helpful to analyze image for color improvement, feature enrichment, enrichment of spatial resolution also the fusion of dissimilar data sets. In this method, spectral information is generally reflected over the hue and the saturation. The objective is guaranteed the spectral information and manipulating the complete information of high spatial resolution for the high-resolution and multispectral remote sensing images, so the fusion is more sufficient for dealing in IHS space [8].

\subsection{Hilbert Transform}

Hilbert transform (HT) is a logical tool that is helpful to represent certain types of signals such as band pass signals and also helpful for several types of modulation techniques like series side band, AM modulation. In HT transform no modification in domain is required that's why HT transform is different from another transform that is used in signal processing. With the help of HT if the contributed signal is available in time domain then the resultant signal is also in time domain only [9]. This characteristic of HT is also correct for the signal that is in frequency domain helps to decrease the complexity. The universal form of the HT in equation (2)

$$
x^{\wedge}(t)=\frac{1}{\pi} \int_{-\infty}^{\infty} \frac{x(\tau)}{t-\tau} d \tau
$$

Where $x^{\wedge}(t)$ is the Hilbert transform of $x(t)$.

The HT includes the convolution of the contributed signal and the resultant output. The Linear Filters attained the transfer function as it assures superposition principle and that will be only presented in transfer function form. All the frequency components are phase shifted by $-\pi / 2$ radians with the help of linear filter [10]. For all the frequencies the magnitude characteristics of the filter are 1 as the actual signals have affirmative and negative frequencies. Phase exchange of the existent signal happens due to double shift of $90^{\circ}$ phase introduces by $\mathrm{HT}$ and a total of $180^{\circ}$ phase shift occurs.

For the inverse Hilbert transform (IHT) as shown in equation (3).

$x(t)=-\frac{1}{\pi} \int_{-\infty}^{\infty} \frac{x^{\wedge}(\tau)}{t-\tau} d \tau$

The equation (1) and (2) demonstrates that HT is a linear operator. The input and the output $\mathrm{x}(\mathrm{t})$ is phrased as Hilbert transform pairs.

\section{LITERATURE SURVEY}

Mozhdeh Haddadpour et al. [1] proposed the two dimensional Hilbert transform (2-D HT) and Intensity Hue Saturation (IHS) method. The MRI and PET images were merged which were used as input images. To calculate the action of the proposed technique the three evaluation metrics were used that were Average Gradient $\left(\mathrm{AG}_{\mathrm{k}}\right)$, the lowest Discrepancy $\left(D_{k}\right)$ and Overall Performance (O.P). The simulation results had demonstrated that the proposed technique could conserve the spatial and spectral features of input images.

Cheng-I Chen [2] had discussed on the basis of IntensityHue-Saturation model and log-Gabor wavelet transform the author proposed a novel fusion technique was proposed to efficiently detect or diagnose a likely disease by doctors. First of all, log-Gabor wavelet transform with suitable decomposition scales subdivided into the MRI image and the intensity component of PET image. After that three rules were employed that were maximum selection' fusion rule for the high-frequency sub-band and visibility measure for the lowfrequency sub-band and two-stage fusion rule based on weighted-averaging scheme. At last by using reverse logGabor wavelet transform to the merged high-and lowfrequency sub-bands a novel intensity component was acquired and to acquire a fused color image, original hue and saturation components of PET image were transformed. In these merged images both of the anatomical structures and color changes were provided with efficiently decreased color distortion. The simulation results were on twelve data sets that include normal axial, normal coronal and Alzheimer's disease brain images showed that the fusion method outpaces framelet-transform-based method both visually and quantitatively.

Mehdi Sefidgar Dilmaghani et al. [3] proposed a novel method based on bi-dimensional empirical mode decomposition (BEMD) was proposed in this paper. This proposed method was used after applying IHS method to decompose the MRI and the intensity component of PET image. After this, useful details of both images were invented and extras were removed. The efficiency of this technique and the quality of input and output images was verified by using visual and numerical evaluation techniques. The proposed method offered much better results than PCA and wavelet fusion method. 
Behzad Kalafje Nobariyan et al. [4] has discussed with the help of MRI and image fusion method the Single Photon Emission Computed Tomography (SPECT) was developed to acquire the fused image having functional and anatomical information. The tissue brain anatomy was demonstrated by MRI image. Without functional information, MRI had high spatial resolution whereas SPECT had low spatial resolution that demonstrated the brain function. So the fusion of both (SPECT and MRI) images offered a high spatial resolution. With the preferred specifications the fused image contained spatial and spectral distortions. The proposed method conserves both the spatial and spectral information but reduces the distortion of fused images comparative to other methods.

Maruturi Haribabu et al. [5] had proposed that MRI-PET medical image fusion has key scientific significance. After registration, the medical image fusion was the relevant step i.e. an integrative display technique of two images. Where the PET image demonstrated the brain function having less spatial resolution there MRI image demonstrated the brain tissue anatomy that attains no functional information. The fused image that acquired functional information and spatial characteristics was perfect without any spatial distortion and color distortion. On the basis of average and spatial frequency technique the DWT coefficients of MRI-PET intensity principles were fused. The proposed method was very simple and could be implemented in actual time applications.

Changtao He et al. [6] reviewed a survey that the conventional methods had been proposed for fusing multimodal image formation but for a particular function the conventional methods had its limits. It was examined that Intensity-hue-saturation (IHS) transform and Principal component analysis (PCA) could conserve much spatial aspect and more essential functional information with no color distortion. In this paper, to enhance the fused image superiority, the benefits of IHS and PCA fusion techniques were integrated. The simulation results of the proposed work had shown that the fusion quality had improved significantly as compared to other fusion methods that included PCA, DWT, BT.

Te-Ming Tu et al. [7] had shown that the modern image fusion techniques, that were IHS, PCA, BT and WT, face color distortion had troubled with fused images. In this paper it was examined that the color distortion problem occurred due to the change of saturation throughout the fusion practice. The simulation results had shown the results for distinct image fusion methods.

Sabalan Daneshvar et al. [8] introduced a paradigm that could combine the benefits of IHS and RIM fusion techniques to advance the functional and spatial information content. As the intensity-hue-saturation (IHS) algorithm and the retinainspired model (RIM) fusion method could conserve much spatial aspect and more functional information content. The simulation results had demonstrated that the proposed paradigm enhanced the fusion superiority (in terms of entropy, mutual information, discrepancy, and average gradient) compared to the other fusion methods like IHS, BT, discrete wavelet transforms (DWT), à-trous wavelet and RIM.

Meenu Manchanda et al. [9] had proposed a new technique of multimodal medical image fusion using fuzzy-transform (FTR). The fusion on the basis of FTR was facilitated in conservation with the efficient transfer of complete information presented in input images into a fused image. In this paper the proposed method was compared and experimented with other traditional methods of fusion to calculate the better presentation. So the simulation results had shown that the proposed fusion paradigm was more efficient rather than other methods of fusion and it also had generated better results.

\section{CONCLUSION AND FUTURE SCOPE}

As the technology grows, the most of the decision making system relies on digital form of data. The images are also a form of digital data and the process of image based decision making system is adopted by various domains nowadays. The decision making is a crucial process and it requires having most of the accuracy thus the concept of image processing comes to the existence, as the destructive images or noisy images cannot results to effective decision. In image processing, the images are processed to enhance their quality. Image fusion is also a part of image processing. The aim of Image fusion is to merge two or more than two corrupted input images to attain a single output image with all required information. So the image fusion is used to improve or advance the superiority of an image. Several fusion mechanisms like PCA, SWT, DWT, DCT etc. are used to achieve this goal. After analyzing the earlier work, it is concluded that by employing different optimization techniques the optimized outputs are required to be fused.

In future more enhancements are possible to perform the advancements in the image fusion mechanism.

\section{REFERENCES}

[1] Mozhdeh Haddadpour, Sabalan Daneshvar, Hadi Seyedarabi, "PET and MRI image fusion based on combination of 2-D Hilbert transform and IHS method", Biomedical Journal, Volume 40, Issue 4, Pp 219-225, 2017.

[2] Cheng-I Chen, "Fusion of PET and MR Brain Images Based on IHS and Log-Gabor Transforms", IEEE Sensors Journal, Volume: 17, Issue: 21, Pp 6995 - 7010, 2017

[3] Mehdi Sefidgar Dilmaghani; Sabalan Daneshvar; Mehdy Dousty, "A new MRI and PET image fusion algorithm based on BEMD and HIS methods", (ICEE), Pp 118-121, 2017

[4] Behzad Kalafje Nobariyan; Sabalan Daneshvar; Mehdi Hosseinzadeh, "Fusion of SPECT and MRI images using back and fore ground information", (MVIP), Pp 227-231, 2013

[5] Maruturi Haribabu; Ch. Hima Bindu; K. Satya Prasad, "Multimodal Medical Image Fusion of MRI-PET Using Wavelet Transform", International Conference on Advances in Mobile Network, Communication and Its Applications, Pp 127 - 130, 2012

[6] Changtao He, Quanxi Liu, Hongliang Li, Haixu Wang, "Multimodal medical image fusion based on IHS and PCA", Procedia Engineering, Volume 7, Pp 280-285, 2010.

[7] Te-Ming Tu, Shun-Chi Su, Hsuen-Chyun Shyu, Ping S. Huan,"A new look at HIS like image fusion methods",Information Fusion, Volum e 2, Issue 3, Pp 177-186, 2001. 
[8] Sabalan Daneshvar, Hassan Ghassemian, "MRI and PET image fusion by combining IHS and retina-inspired models", Information Fusion, Volume 11, Issue 2, Pp 114-123, 2010.

[9] Meenu Manchanda, Rajiv Sharma, "A novel method of multimodal medical image fusion using fuzzy transform", Journal of Visual Communication and Image Representation, Volume 40, Part A, Pp 197$217,2016$.

[10] Yu Liu, Xun Chen, Zengfu Wang, Z. Jane Wang, Xuesong Wang, "Deep learning for pixellevel image fusion: Recent advances and future prospects", Information Fusion, Volume 42, Pp 158173,2018 . 